\title{
Der Spinabschneideparameter für einige mittelschwere Kerne aus Neutronenreaktionen
}

\author{
M. Bormann, F. Dreyer, U. Seebeck u. W. Voigts \\ I. Institut für Experimentalphysik, Universität Hamburg \\ (Z. Naturforschg. 21 a, 988-994 [1966]; eingegangen am 7. März 1966) \\ Professor Dr. W. Gentner zum 60. Geburtstag gewidmet
}

\begin{abstract}
In der Kernniveaudichte nach dem F FrMI-Gas-Modell wird die Abhängigkeit der Dichte der Kernniveaus vom Drehimpuls des Kerns wesentlich durch den Spinabschneideparameter $\sigma$ bestimmt. Dieser kann unter anderem aus den Winkelverteilungen der emittierten Teilchen und aus dem Verhältnis isomerer Wirkungsquerschnitte für Compoundkernreaktionen erhalten werden, die sich nach der statistischen Theorie der Kernreaktionen beschreiben lassen. Für einige Kernreaktionen schneller Neutronen wurden derartige Meßdaten ermittelt und daraus Spinabschneideparameter $\sigma$ für die Kerne $\mathrm{Ar}^{37}, \mathrm{Co}^{58}, \mathrm{Ni}^{61}, \mathrm{Ge}^{75}$ und $\mathrm{Se}^{73}$ abgeleitet. Die Ergebnisse werden verglichen mit entsprechenden Angaben anderer Arbeiten für mittelschwere Kerne und mit den Voraussagen des Fermi-Gas-Modells.
\end{abstract}

Die Dichte der Energiezustände angeregter Kerne spielt in der Kernphysik eine wesentliche Rolle. Sie ist z. B. von zentraler Bedeutung bei der Interpretation des großen Bereichs der Compoundkernreaktionen durch die statistische Theorie, und durch sie wird nach der Theorie der Kernspaltung auch die Massenverteilung der Spaltbruchstücke wesentlich bestimmt. Häufig interessiert dabei nicht nur die gesamte bei der Anregungsenergie $E$ vorliegende Niveaudichte $\omega(E)$ eines Kerns, sondern auch die Dichte $\omega(E, J)$ der Niveaus mit einem bestimmten Drehimpuls $J$. Im freien FERMI-Gas-Modell für den Kern folgt unter der Annahme der statistischen Verteilung der Drehimpulse auf die Einteilchen-Zustände des Fermi-Gases für $\omega(E, J)$ die Beziehung ${ }^{1}$

$$
\begin{aligned}
\omega(E, J)=C(2 J+1) E^{-2} \exp \{ & -J(J+1) / 2 \sigma^{2} \\
& +2 \sqrt{a} E\} .
\end{aligned}
$$

Hierin sind $C$ und $a$ nur noch von der Massenzahl $A$ des Kerns abhängig. Für den Spinabschneideparameter $\sigma$ ergibt sich der Zusammenhang

$$
\sigma^{2}=\left(\mathcal{T}_{0} T\right) / \hbar^{2}
$$

mit dem Kernträgheitsmoment $\mathcal{T}_{0}$ und der Kerntemperatur $T$. Das Kernträgheitsmoment $\mathcal{T}_{0}$ eines als freies Fermi-Gas betrachteten Kerns mit dem Radius $R$ und der Massenzahl $A$ ist gleich dem des als starrer Körper angesehenen Kerns, nämlich

$$
\mathcal{T}_{0}=(2 / 5) m A R^{2},
$$

1 T. Ericson, Phil. Mag. Suppl. 9, 425 [1960].

2 M. Bormann u. H. Neuert, Fortschr. Phys. 11, 277 [1963]. wobei $m$ die Nukleonenmasse bedeutet. Für die Kerntemperatur folgt in guter Näherung

$$
E=a T^{2}-4 T \text {. }
$$

Nach (1) wird somit die Abhängigkeit der Niveaudichte vom Drehimpuls $J$ wesentlich durch den Parameter $\sigma$ bestimmt, während die Abhängigkeit von der Anregungsenergie $E$ hauptsächlich durch den Parameter $a$ festgelegt wird. Der Paarungseffekt in der Kernniveaudichte läßt sich im allgemeinen befriedigend durch die Ersetzung von $E$ durch $U=E-\delta$ in (1) berücksichtigen, wobei $\delta$ die Paarungsenergie des betrachteten Kerns bedeutet (siehe z. B. ${ }^{1,2}$ ).

Der Niveaudichteparameter $a$ kann insbesondere aus experimentellen Verdampfungsspektren der in Compoundkernreaktionen emittierten Teilchen oder aus Neutroneneinfangresonanzen bestimmt werden ${ }^{2,3}$. Zur Ermittlung empirischer Daten über den Spinabschneideparameter $\sigma$ sind in der Literatur im wesentlichen drei Verfahren bekannt. Die direkte Methode der Auszählung der Kernniveaus mit bestimmtem Drehimpuls $J$ für einen gewissen Bereich der Anregungsenergie und Überprüfung des Ergebnisses mit (1) ist praktisch nur sehr unvollkommen durchzuführen. Es gelingt experimentell nicht, alle Niveaus eines Kerns wesentlich oberhalb des Grundzustandes aufzulösen und deren Spins vollständig zu bestimmen. Untersuchungen nach diesem Verfahren

3 E. Erba, U. Facchini u. E. Saetta-Menichella, Nuovo Cim. 22, 1237 [1961]. 
können daher nur Abschätzungen für $\sigma$ liefern und wurden durchgeführt z. B. in ${ }^{4-7}$.

Häufiger sind in der Literatur Aussagen über $\sigma$ aus den Winkelverteilungen der Reaktionsprodukte von Compoundkernreaktionen mit Hilfe der statistischen Theorie ermittelt worden. Diese Theorie sagt für die Winkelverteilung der verdampften Teilchen im Schwerpunktsystem eine Symmetrie um $90^{\circ}$ voraus mit einem Minimum bei diesem Winkel ${ }^{8}$. Für den Fall, daß der Bahndrehimpuls $l_{\mathrm{b}}$ des in der Reaktion $A(\mathrm{a}, \mathrm{b}) B$ emittierten Teilchens $\mathrm{b}$ nur schwach mit dem Spin $J_{\mathrm{c}}$ des Compoundkerns gekoppelt ist, folgt auch nur eine schwache Anisotropie für die Winkelverteilung der Teilchen b. Die Winkelverteilung kann dann näherungsweise halbklassisch berechnet werden und führt $\mathrm{zu}^{1,9,10}$

$$
W(\Theta)=1+\frac{\left\langle J_{\mathrm{c}}{ }^{2}\right\rangle\left\langle l_{\mathrm{b}}{ }^{2}\right\rangle}{12 \sigma^{4}} P_{2}(\cos \Theta) .
$$

$W(\Theta)$ ist die relative Winkelverteilung, $P_{2}(\cos \Theta)$ das zweite Legendresche Polynom, $\left\langle J_{\mathrm{c}}{ }^{2}\right\rangle$ der Mittelwert des Spinquadrats des Compoundkerns und $\left\langle l_{\mathrm{b}}{ }^{2}\right\rangle$ das mittlere Bahndrehimpulsquadrat der emittierten Teilchen b. Durch Vergleich von (5) mit entsprechenden experimentellen Daten kann somit $\sigma$ ermittelt werden.

Die umfangreichsten Informationen über $\sigma$ sind bisher jedoch nach der von Huizenga und Vanden${ }_{\text {Bosch }}{ }^{11,12}$ vorgeschlagenen Methode aus dem Verhältnis isomerer Wirkungsquerschnitte von Compoundkernreaktionen erhalten worden. Viele Kerne besitzen über dem Grundzustand ein metastabiles Niveau, dessen Drehimpuls wesentlich von dem des Grundzustands verschieden ist. Verläuft eine Reaktion zu hochangeregten Zuständen eines solchen Kerns, so können die nachfolgenden $\gamma$-Kaskaden entweder auf dem metastabilen oder auf dem Grundzustand des Kerns enden. Die Häufigkeit, mit der die beiden isomeren Zustände in der betrachteten Reaktion bevölkert werden, hängt unter anderem von der Verteilung der Drehimpulse auf die angeregten Kernzustände und damit nach (1) vom Spinab-

4 T. ERIcson, Nucl.Phys. 11, 481 [1959].

5 C. T. Hibdon, Phys. Rev. 114, 179 [1959] und Phys. Rev. 122, 1235 [1961].

6 J. H. Carver u. G. A. Jones, Nucl. Phys. 19, 184 [1960].

7 H. K. Vonach, Habil.-Schrift, Techn. Hochschule, München 1965.

8 A. C. Douglas u. N. MacDonald, Nucl. Phys. 13, 382 [1959].

9 T. Ericson, Nucl. Phys. 17, 250 [1960]. schneideparameter $\sigma$ ab. Aus der Analyse experimenteller Werte für das Verhältnis der Wirkungsquerschnitte, mit denen eine Reaktion zum Grund- bzw. metastabilen Zustand des Restkerns führt, können daher nach dem Verfahren von Huizenga und VANDENBosch Aussagen über $\sigma$ gewonnen werden.

In der vorliegenden Arbeit wurden nach den beiden letztgenannten Verfahren Werte für $\sigma$ einiger mittelschwerer Kerne aus entsprechenden Meßdaten für Neutronenreaktionen bestimmt. Dazu wurden einmal die Winkelverteilungen der $\alpha$-Teilchen aus den mit 14,1 MeV-Neutronen ausgelösten Reaktionen $\mathrm{Ca}^{40}(\mathrm{n}, \alpha) \mathrm{Ar}^{37}$ und $\mathrm{Zn}^{64}(\mathrm{n}, \alpha) \mathrm{Ni}^{66}$ gemessen. Zum anderen wurde das Verhältnis der Wirkungsquerschnitte für die Bildung eines isomeren Restkernzustands zum totalen Wirkungsquerschnitt für die Reaktionen $\mathrm{Co}^{59}(\mathrm{n}, 2 \mathrm{n}) \mathrm{Co}^{58}$ und $\mathrm{Se}^{74}(\mathrm{n}, 2 \mathrm{n}) \mathrm{Se}^{73}$ als Funktion der Neutronenenergie im Energiebereich 12,6 bis 19,6 MeV sowie für die Reaktionen $\mathrm{Ni}^{58}(\mathrm{n}, \mathrm{p}) \mathrm{Co}^{58}$ bei $13,0 \mathrm{MeV}$ und $\mathrm{As}^{75}(\mathrm{n}, \mathrm{p}) \mathrm{Ge}^{75}$ bei $14,1 \mathrm{MeV}$ Neutronenenergie ermittelt.

\section{Experimentelle Methoden}

Die Winkelverteilungen der $\alpha$-Teilchen wurden mit Hilfe eines dünnen CsJ(Tl)-Szintillationskristalls gemessen unter Anwendung der Methode der Impulsformdiskriminierung zur Unterscheidung der $\alpha$-Teilchen von anderer, als Untergrund auftretender Strahlung. Die gesamte Reaktionskammer sowie die elektronischen und weiteren meßtechnischen Einzelheiten wurden in ${ }^{13}$ bereits ausführlich beschrieben. Als Targets wurden Folien von $(2 \times 2) \mathrm{cm}^{2}$ Fläche verwandt. Im Fall des $\mathrm{Ca}$ bestand die Folie aus natürlichem $\mathrm{Ca}\left(97 \% \mathrm{Ca}^{40}\right)$ und hatte eine Dicke von $6 \mathrm{mg} / \mathrm{cm}^{2}$. Die Zn-Folie bestand zu $96 \%$ aus $\mathrm{Zn}^{64}$ und ihre Dicke betrug $4 \mathrm{~cm} / \mathrm{cm}^{2}$ *.

Die Messung der übrigen Wirkungsquerschnitte erfolgte nach der Aktivierungsmethode. Die untersuchten Reaktionen führten alle zu radioaktiven Restkernen, die in ihrem Zerfall Positronen emittieren. Daher wurden in allen Reaktionen mit Ausnahme der Reaktion $\mathrm{As}^{75}(\mathrm{n}, \mathrm{p}) \mathrm{Ge}^{75}$ die erzeugten Aktivitäten durch koinzidenten Nachweis der beiden Gamma-Quanten aus der Positronenvernichtung nachgewiesen. Hierzu diente ein Koinzidenzspektrometer, das aus zwei NaJ (Tl)-Kristallen von $7,5 \mathrm{~cm}$ Durchmesser und Höhe bestand, und

10 T. Ericson u. V. Strutinski, Nucl. Phys. 8, 284 [1958] und Nucl. Phys. 9, 689 [1958].

11 J. R. Huizenga u. R. Vandenbosch, Phys. Rev. 120, 1305 [1960].

12 W. L. Hafner, J. R. Huizenga u. R. Vandenbosch, Report ANL-6662 (1962).

13 U. Seebeck u. M. Bormann, Nucl. Phys. 68, 387 [1965].

* Die $\mathrm{Zn}^{64}$-Folie wurde bezogen von der Electromagnetic Seperation Group, AERE, Harwell, England. 
das eine Auflösungszeit von $5 \cdot 10^{-8}$ sec besaß. Die Ansprechwahrscheinlichkeit der Meßanordnung wurde experimentell durch Ausmessen von Vergleichsreaktionen mit bekanntem Wirkungsquerschnitt $\left(\mathrm{Cu}^{63}(\mathrm{n}, 2 \mathrm{n}) \mathrm{Cu}^{62}\right.$ und $\mathrm{F}^{\mathbf{1 9}}(\mathrm{n}, 2 \mathrm{n}) \mathrm{F}^{\mathbf{1 8}}$ bei $14,1 \mathrm{MeV}$ Neutronenenergie, $\left.\sigma_{\mathrm{n}, 2 \mathrm{n}}\left(\mathrm{Cu}^{63}\right)=480 \pm 38 \mathrm{mb}, \quad \sigma_{\mathrm{n}, 2 \mathrm{n}}\left(\mathrm{F}^{19}\right)=41,2 \pm 2,5 \mathrm{mb}\right)$ bestimmt. Im Fall der Reaktion $\operatorname{As}^{75}(n, p) \mathrm{Ge}^{75}$ wurde die gesamte Gamma-Aktivität des Restkerns $\mathrm{Ge}^{75} \mathrm{mit}$ einem $\mathrm{NaJ}(\mathrm{Tl})$-Bohrlochspektrometer von $7,5 \mathrm{~cm}$ Durchmesser und $5 \mathrm{~cm}$ Höhe registriert, wobei zur Messung alle Gamma-Quanten zugelassen wurden, die in dem Kristall einen Energieverlust von mehr als $50 \mathrm{keV}$ erlitten. Die Ansprechwahrscheinlichkeit des Spektrometers für diese Diskriminatorschwelle und die Gamma-Strahlung des $\mathrm{Ge}^{75}$ wurde berechnet. Weitere Einzelheiten über die Meßapparaturen zur Aktivitätsmessung sind in ${ }^{14}$ angegeben.

Als Neutronenquelle diente die Reaktion $\mathrm{T}(\mathrm{d}, \mathrm{n}) \mathrm{He}^{4}$, die zur Erzeugung von 14 MeV-Neutronen mit $150 \mathrm{keV}$ Deuteronen und von Neutronen im Energiebereich 12,6 bis $19,6 \mathrm{MeV}$ mit $3 \mathrm{MeV}$-Deuteronen in einem dünnen Titan-Tritium-Target ausgelöst wurde. Im letzteren Fall konnte die Neutronenenergie durch Verändern des Beobachtungswinkels variiert werden. Alle Wirkungsquerschnitte wurden bei $14,1 \mathrm{MeV}$ Neutronenenergie absolut gemessen, hierzu wurde der Neutronenfluß mit einem Stilben-Rückstoßprotonen-Spektrometer mit einer Genauigkeit von 4,5\% gemessen. Die Wirkungsquerschnitte bei anderen Neutronenenergien wurden relativ zu den Werten bei 14,1 MeV erhalten. Für die Abhängigkeit der Neutronenintensität vom Beobachtungswinkel im Fall der Neutronenerzeugung mit $3 \mathrm{MeV}$-Deuteronen wurden die entsprechenden Angaben von PAULSEN und Liskien ${ }^{15}$ zugrunde gelegt.

\section{Meßergebnisse und ihre Analyse}

Die unter verschiedenen Winkeln im Schwerpunktsystem erhaltenen Spektren der $\alpha$-Teilchen, die bei Beschuß von $\mathrm{Ca}^{40}$ und $\mathrm{Zn}^{64}$ mit $14,1 \mathrm{MeV}$-Neutronen emittiert werden, sind in Abb. 1 und 2 wiedergegeben. Die Spektren sind aufgetragen in der Form des sog. statistischen Plots, der nach der statistischen Theorie für Verdampfungsspektren nahegelegt wird. Das Spektrum $N\left(\varepsilon_{a}\right)$ der in der Reaktion $A(\mathrm{n}, \alpha) B$ verdampften $\alpha$-Teilchen hat nach dieser Theorie die Form ${ }^{1,2}$

$$
N\left(\varepsilon_{a}\right) \sim \varepsilon_{a} \sigma_{\mathrm{c}}\left(\varepsilon_{a}\right) \omega(E)
$$

mit der $\alpha$-Teilchen-Energie $\varepsilon_{a}$, dem Wirkungsquerschnitt $\sigma_{\mathrm{c}}\left(\varepsilon_{a}\right)$ für die Bildung des Compoundkerns durch die inverse Reaktion und der Niveaudichte

14 M. Bormann, E. Fretwurst, P. Schehka, G. Wrege, H. Büttner, A. Lindner u. H. Meldner, Nucl. Phys. 63, 438 [1965].

15 A. Paulsen u. H. Liskien, Nucl. Phys. 56, 394 [1964].
$\omega(E)$ des Restkerns bei der zu $\varepsilon_{a}$ gehörigen Anregungsenergie $E$. Für $\omega(E)$ wurde nach (1) der Ansatz

$$
\omega(E) \sim(E-\delta)^{-2} \exp (2 \sqrt{a(E-\delta)})
$$

zugrunde gelegt. Dann ergibt sich nach (6) und (7) bei Auftragung des reduzierten Spektrums

$$
N^{\prime}\left(\varepsilon_{a}\right)=\frac{N\left(\varepsilon_{a}\right)(E-\delta)^{2}}{\varepsilon_{a} \cdot \sigma_{\mathrm{c}}\left(\varepsilon_{a}\right)}
$$

als Funktion von $(E-\delta)^{1 / 2}$ eine Gerade mit dem Anstieg $2 \sqrt{a}$. Für $\sigma_{\mathrm{c}}\left(\varepsilon_{a}\right)$ wurden die Werte nach Huizenga und IGo ${ }^{16}$ benutzt und für $\delta$ die Paarungsenergien nach Nemirovsky und.AdamchuK ${ }^{17}$ gewählt. Die Abb. 1 und 2 zeigen für jeweils ein Target und alle Beobachtungswinkel parallele Geraden, deren Anstiege die $a$-Werte für die Restkerne $\mathrm{Ar}^{37} \mathrm{bzw}$. $\mathrm{Ni}^{61}$ ergeben. Bei großen Werten für $E$ (kleine Energien $\varepsilon_{a}$ ) werden teilweise Abweichungen von diesen

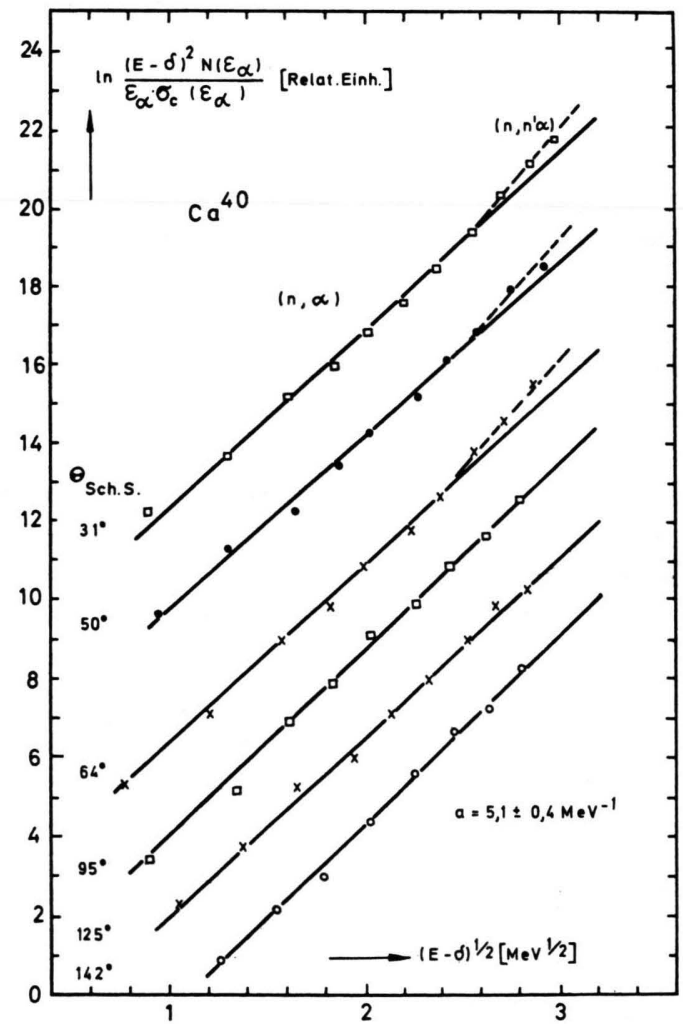

Abb. 1. Statistische Plots der unter verschiedenen Winkeln gemessenen $\alpha$-Teilchen-Spektren aus Kernreaktionen von 14,1 MeV-Neutronen in $\mathrm{Ca}^{40}$.

16 J. R. Huizenga u. G. Igo, Nucl. Phys. 29, 462 [1962] und Report ANL-6373 (1961).

17 P. E. Nemirovsky u. Yn. V. Adamchuk, Nucl. Phys. 39, 551 [1962]. 


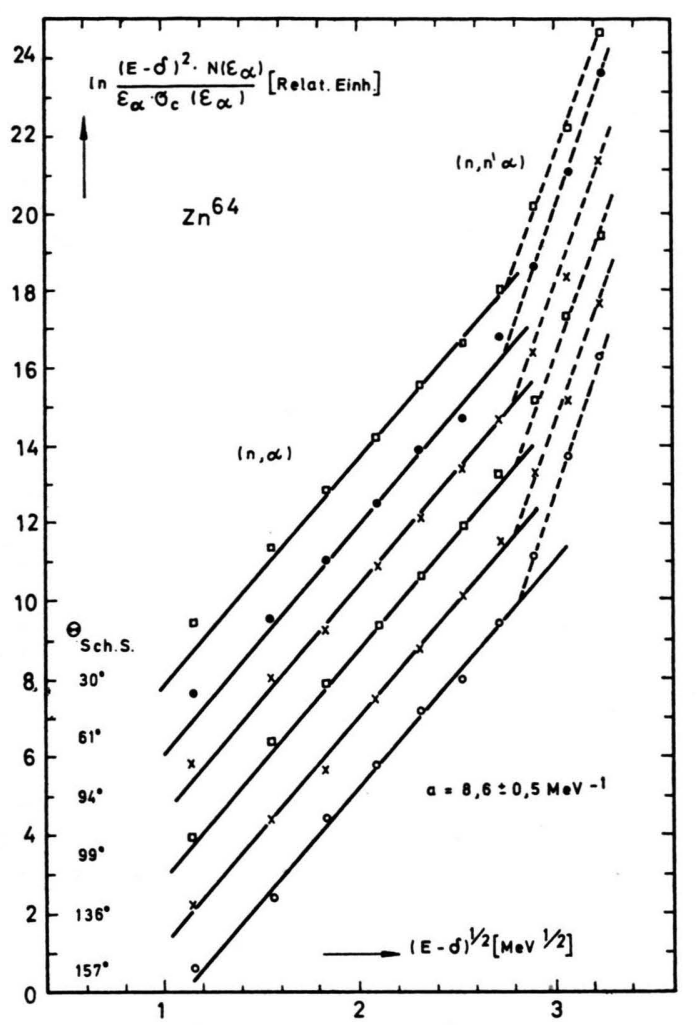

Abb. 2. Statistische Plots der unter verschiedenen Winkeln gemessenen $a$-Teilchen-Spektren aus Kernreaktionen von $14,1 \mathrm{MeV}-$ Neutronen in $\mathrm{Zn}^{64}$.

Geraden beobachtet. Diese Abweichungen werden $\alpha$-Teilchen aus der Reaktion ( $n, n^{\prime} \alpha$ ) zugeschrieben. Die Geraden dagegen werden gebildet durch $\alpha$-Teilchen aus den Reaktionen (n, $\alpha \gamma)$ und (n, $\left.\alpha \mathrm{n}^{\prime}\right)$, die im folgenden zusammen als Reaktion $(n, \alpha)$ bezeichnet werden.

Im Falle des Ca konnten für die Winkel oberhalb $90^{\circ}$ die $\alpha$-Teilchen im niederenergetischen Teil der Spektren nicht mehr vollständig nachgewiesen werden, da hier die Energien teilweise so klein waren, daß die $\alpha$-Teilchen in der Ca-Folie einen zu großen Energieverlust erlitten und unterhalb der Nachweisschwelle der Meßapparatur lagen. Über die $\left(n, n^{\prime} \alpha\right)$. Anteile in diesen Spektren können daher keine Angaben gemacht werden.

Die Spektren wurden gemäß den Abb. 1 und 2 in die Anteile aus den Reaktionen $(n, \alpha)$ und $\left(n, n^{\prime} \alpha\right.$ ) aufgeteilt und jeweils die Gesamtintensitäten in Abb. 3 als Funktion des Winkels aufgetragen. Im Fall des Ca wurden dabei die Spektren aus der (n, a)-Reaktion für Winkel oberhalb $90^{\circ}$ im niederenergetischen Teil gemäß der Geraden im übrigen
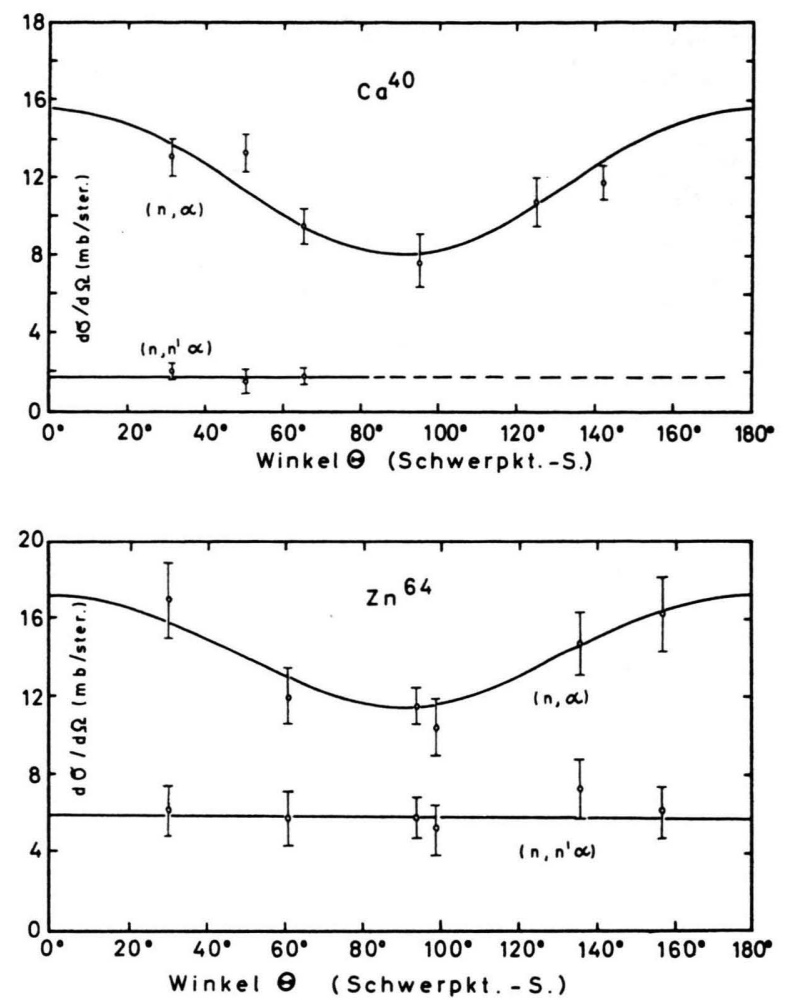

Abb. 3. Winkelverteilungen der $\alpha$-Teilchen aus Kernreaktionen von $14,1 \mathrm{MeV}$-Neutronen in $\mathrm{Ca}^{40}$ und $\mathrm{Zn}^{64}$.

Energiebereich ergänzt. Die Winkelverteilungen sind, wie nach der statistischen Theorie zu erwarten ist, symmetrisch um $90^{\circ}$, die Verteilung für $\mathrm{Zn}^{64}\left(\mathrm{n}, \mathrm{n}^{\prime} \alpha\right)$ ist sogar isotrop. Es wurde angenommen, daß eine solche Isotropie auch für die Winkelverteilung der a-Teilchen aus der Reaktion $\mathrm{Ca}^{40}\left(\mathrm{n}, \mathrm{n}^{\prime} \alpha\right)$ vorliegt.

Die Winkelverteilungen der $(n, \alpha)$-Reaktionen wurden mit der Beziehung (5) nach der statistischen Theorie verglichen. Dabei wurden die mittleren Drehimpulsquadrate $\left\langle J_{\mathrm{c}}{ }^{2}\right\rangle$ und $\left\langle l_{\alpha}{ }^{2}\right\rangle$ gebildet mit den Gewichtsfaktoren

$$
\frac{\left(2 J_{\mathrm{c}}+1\right) T_{l}\left(\varepsilon_{\mathrm{n}}\right)}{\sum_{J_{\mathrm{c}}=0}^{\infty}\left(2 J_{\mathrm{c}}+1\right) T_{l}\left(\varepsilon_{\mathrm{n}}\right)} \quad \text { und } \frac{\left(2 l_{\alpha}+1\right) \overline{T_{l}\left(\varepsilon_{a}\right)}}{\sum_{l \alpha=0}^{\infty}\left(2 l_{\alpha}+1\right) \overline{T_{l}\left(\varepsilon_{a}\right)}} \text {. }
$$

Hier sind $T_{l}\left(\varepsilon_{\mathrm{n}}\right)$ der Transmissionskoeffizient der mit der Einschußenergie $\varepsilon_{\mathrm{n}}$ einfallenden Neutronen mit dem Bahndrehimpuls $l_{\mathrm{n}}$ und $\overline{T_{l}\left(\varepsilon_{a}\right)}$ der über die $\alpha$-Teilchen-Energien gemittelte Transmissionskoeffizient der mit dem Bahndrehimpuls $l_{\alpha}$ emittierten a-Teilchen, wobei der Mittelwert durch Bewichtung mit dem jeweiligen gemessenen Energiespektrum bestimmt worden ist. Die Transmissionskoeffizienten für Neutronen wurden der Arbeit von Meldner und 
LindNer $^{18}$ und die für $\alpha$-Teilchen der Arbeit von Huizenga und IGo ${ }^{16}$ entnommen. Die ausgezogenen Kurven in Abb. 3 stellen die mit diesen mittleren Drehimpulsquadraten erhaltenen besten Anpassungen der Beziehung (5) an die experimentellen Winkelverteilungen dar. Sie ergeben sich für die in Tab. 1 aufgeführten Werte für den Parameter $\sigma$. In dieser Tabelle sind ferner auch noch die Werte für den Parameter $a$ aus den Spektren in Abb. 1 und 2 und die aus den Winkelverteilungen folgenden integrierten Wirkungsquerschnitte zusammengestellt.

\begin{tabular}{|c|c|c|c|}
\hline Reaktion & $a\left(\mathrm{MeV}^{-1}\right)$ & $\sigma(\hbar)$ & $\begin{array}{c}\sigma(\mathrm{n}, \alpha) \equiv \\
\equiv \sigma(\mathrm{n}, \alpha \gamma)+ \\
+\sigma\left(\mathrm{n}, \alpha \mathrm{n}^{\prime}\right)\end{array}$ \\
\hline $\mathrm{Ca}^{40}(\mathrm{n}, \alpha) \mathrm{Ar}^{37}$ & $5,1 \pm 0,4$ & $2,8 \pm 0,3$ & $138 \pm 20 \mathrm{mb}$ \\
$\mathrm{Zn}^{64}(\mathrm{n}, \alpha) \mathrm{Ni}^{61}$ & $8,6 \pm 0,5$ & $3,2 \pm 0,3$ & $168 \pm 16 \mathrm{mb}$ \\
\hline
\end{tabular}

Tab. 1.

Für die (n, $\left.n^{\prime} \alpha\right)$-Reaktion ergeben sich als integrierte Wirkungsquerschnitte $23 \pm 6 \mathrm{mb}$ für $\mathrm{Ca}^{40}$ (unter Voraussetzung der oben angegebenen Annahme über den
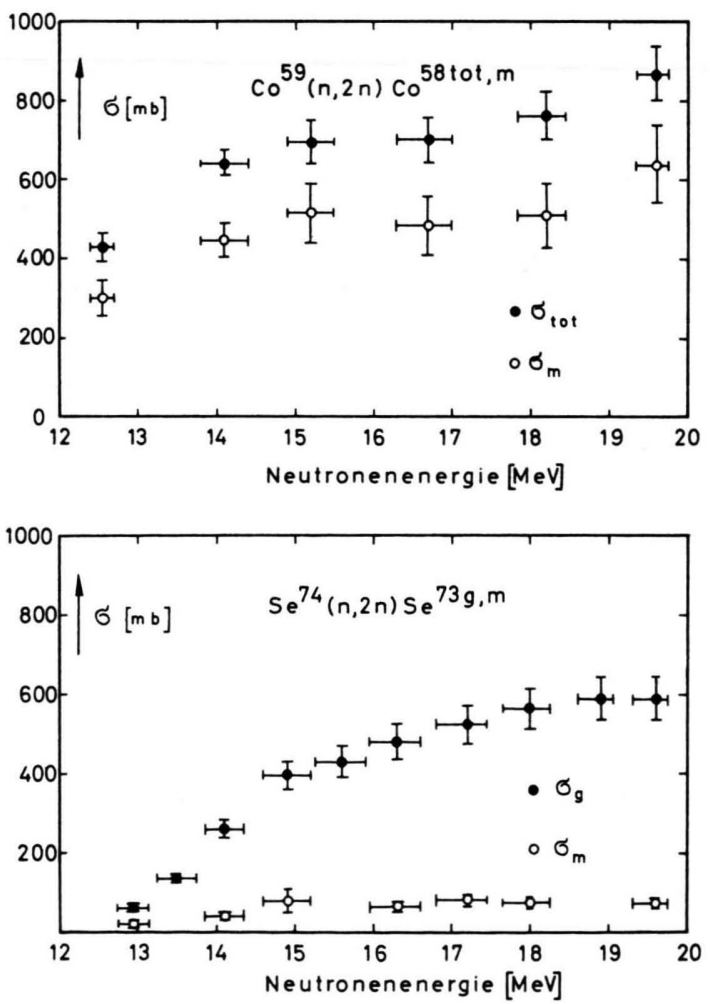

Abb. 4. Anregungsfunktionen für die Reaktionen $\mathrm{Co}^{59}(\mathrm{n}, 2 \mathrm{n}) \mathrm{Co}^{58}$ tot,m und $\mathrm{Se}^{74}(\mathrm{n}, 2 \mathrm{n}) \mathrm{Se}^{73} \mathrm{~g}, \mathrm{~m}$.

18 H. Meldner u. A. Lindner, Z. Phys. 180, 362 [1964].

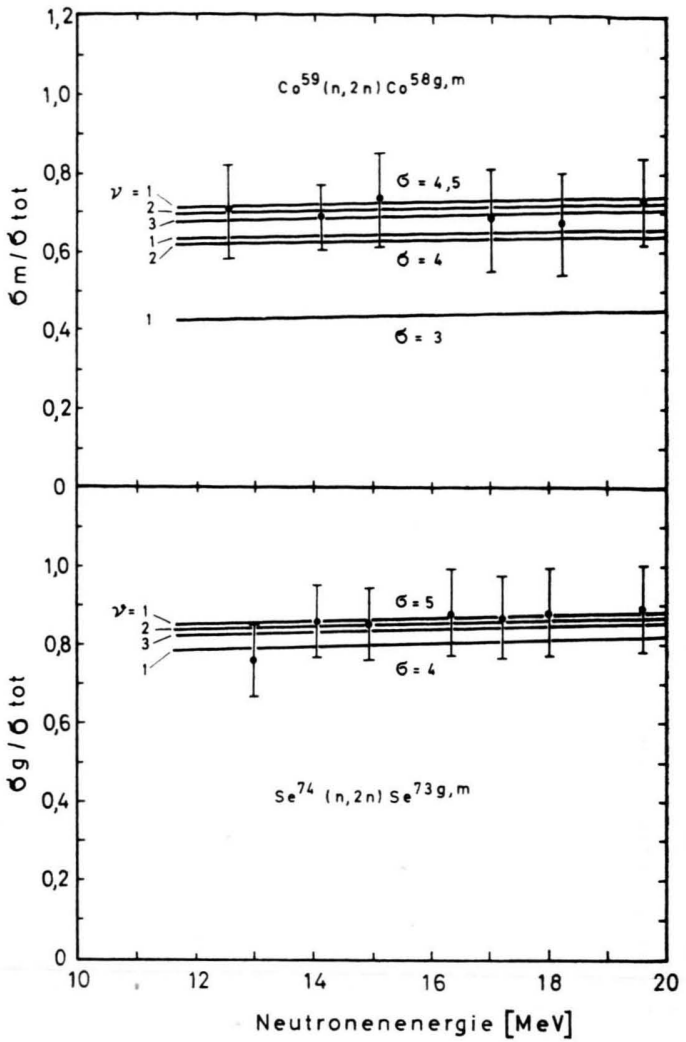

Abb. 5. Verhältnis der isomeren Wirkungsquerschnitte für die Reaktionen $\mathrm{Co}^{59}(\mathrm{n}, 2 \mathrm{n}) \mathrm{Co}^{58} \mathrm{~m}$, tot und $\mathrm{Se}^{74}(\mathrm{n}, 2 \mathrm{n}) \mathrm{Se}^{73} \mathrm{~g}$, tot und Vergleich mit der Theorie von Huizenga und Vandenbosch.

Verlauf der Winkelverteilung für die $\alpha$-Teilchen aus $\operatorname{der}\left(\mathrm{n}, \mathrm{n}^{\prime} \alpha\right)$-Reaktion) und $73 \pm 7 \mathrm{mb}$ für $\mathrm{Zn}^{64}$.

Aus der Analyse des zeitlichen Verlaufs der Aktivität des in der Reaktion $\mathrm{Co}^{59}(\mathrm{n}, 2 \mathrm{n}) \mathrm{Co}^{58}$ erzeugten $\mathrm{Co}^{58}$ wurde der totale Wirkungsquerschnitt $\sigma_{\text {tot }}$ für diese Reaktion und der Wirkungsquerschnitt $\sigma_{\mathrm{m}}$ für die Bildung des Isomers $\mathrm{Co}^{58 \mathrm{~m}}$ bestimmt. Im Falle der Reaktion $\mathrm{Se}^{74}(\mathrm{n}, 2 \mathrm{n}) \mathrm{Se}^{73}$ wurden die Wirkungsquerschnitte $\sigma_{\mathrm{g}}$ und $\sigma_{\mathrm{m}}$ für die Bildung der Isomere $\mathrm{Se}^{73 \mathrm{~g}}$ und $\mathrm{Se}^{73 \mathrm{~m}}$ gemessen. Die Wirkungsquerschnitte sind als Funktion der Neutronenenergie in Abb. 4 wiedergegeben. Abb. 5 zeigt die Verhältnisse $\sigma_{\mathrm{m}} / \sigma_{\text {tot }}$ bzw. $\sigma_{\mathrm{g}} / \sigma_{\text {tot }}$ für die beiden Targetkerne. Hier sind gleichzeitig die Ergebnisse verschiedener Berechnungen nach der Methode von Huizenga und Vandenвозсн unter Verwendung des Niveaudichteansatzes (1) und für verschiedene Parameter $v$ mit eingetragen. Für den Parameter $a$ wurden Werte nach ERBA et al. ${ }^{3}$ gewählt. Der in Abb. 5 auftretende Parameter $v$ bezeichnet die Zahl der Dipol- $\gamma$-Quanten, die der angeregte Restkern emittiert, bevor er in 
einem letzten $\gamma$-Übergang in den Grund- oder metastabilen Zustand zerfällt. Für $v$ kann nach ${ }^{19}$ aus der mittleren Energie der $\gamma$-Quanten und der Anregungsenergie des Restkerns eine obere Grenze abgeschätzt werden. Die theoretischen Berechnungen der isomeren Wirkungsquerschnittsverhältnisse hängen jedoch nur schwach von dem Parameter $v$ ab.

Im Fall der Reaktion $\mathrm{Ni}^{58}(\mathrm{n}, \mathrm{p}) \mathrm{Co}^{58}$ wurden folgende Ergebnisse für $13,0 \mathrm{MeV}$ Neutronenenergie erhalten:

$$
\begin{aligned}
\sigma_{\text {tot }} & =532 \pm 44 \mathrm{mb}, \\
\sigma_{\mathrm{m}} & =305 \pm 26 \mathrm{mb}, \\
\sigma_{\mathrm{m}} / \sigma_{\text {tot }} & =0,57 \pm 0,07 .
\end{aligned}
$$

Für die Reaktion $\mathrm{As}^{75}$ (n, p) $\mathrm{Ge}^{75}$ lauten für 14,1 MeV Neutronenenergie die entsprechenden Ergebnisse:

$$
\begin{aligned}
\sigma_{\text {tot }} & =20,3 \pm 1,6 \mathrm{mb}, \\
\sigma_{\mathrm{m}} & =18,6 \pm 1,5 \mathrm{mb}, \\
\sigma_{\mathrm{m}} / \sigma_{\text {tot }} & =0,92 \pm 0,10 .
\end{aligned}
$$

Bei der Berechnung von $\sigma_{\mathrm{m}} / \sigma_{\text {tot }}$ für die beiden letzten Reaktionen wurde das Verfahren von Huizenga und VANDEnbosch entsprechend dem Vorgehen von NeED ${ }^{20}$ für Reaktionen mit Emission von geladenen Teilchen ergänzt. Als Transmissionskoeffizienten für Neutronen und Protonen wurden dabei wieder die Werte nach Meldner und Lindner ${ }^{18}$ herangezogen. Die Werte für $\sigma$, die die beste Anpassung der theoretischen Verhältnisse der isomeren Wirkungsquerschnitte an die entsprechenden experimentellen Da- ten dieser Arbeit ergaben, sind in Tab. 2 zusammengestellt.

\section{Diskussion}

In dieser Arbeit ist, wie in den meisten entsprechenden Arbeiten in der Literatur, die Energieabhängigkeit von $\sigma$ bei der Analyse der Meßdaten vernachlässigt worden. Die angegebenen Werte für $\sigma$ sind daher als Mittelwerte für den Anregungsenergiebereich von etwa 5 bis $10 \mathrm{MeV}$ anzusehen, in dem die Restkerne bei den hier untersuchten Reaktionen hauptsächlich angeregt worden sind. Aus (2) und (4) folgt jedoch, daß $\sigma$ nur etwa wie $E^{1 / 4}$ mit der Anregungsenergie $E$ variiert, so $\mathrm{da} \beta$ in dem hier betrachteten, relativ engen Anregungsenergiebereich sowieso nur eine schwache Veränderung von $\sigma$ mit $E$ zu erwarten ist. In Abb. 6 sind die in dieser Arbeit erhaltenen Werte für $\sigma$ zusammen mit entsprechenden Ergebnissen anderer Autoren für Kerne im Massenbereich $A=20-80$ aufgetragen. Die Daten beziehen sich alle etwa auf den obengenannten Bereich der Anregungsenergie und sollten daher miteinander verglichen werden können. Die Werte für $\sigma$ wurden folgenden Arbeiten entnommen: Ergebnisse aus Niveauauszählungen nach ${ }^{4-7}$, aus Winkelverteilungen nach $7-10,13,21-24$ und aus Verhältnissen isomerer Wirkungsquerschnitte nach ${ }^{25-32}$. Wie Abb. 6 erkennen läßt, stimmen die in der vorliegenden Arbeit erhaltenen Werte für den Spinabschneide-

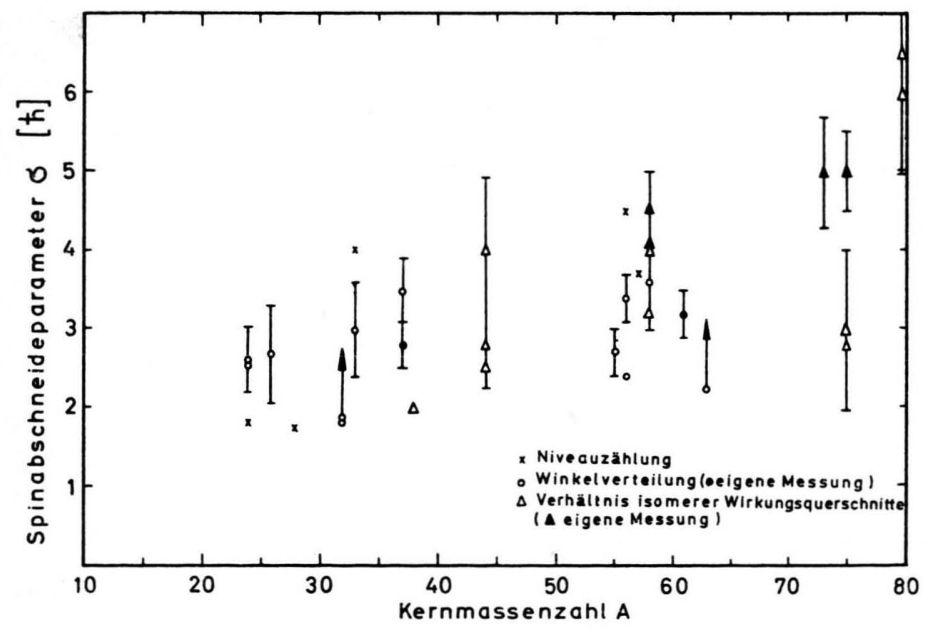

\begin{tabular}{|c|c|r|}
\hline Reaktion & Restkern & \multicolumn{1}{|c|}{$\sigma(\hbar)$} \\
\hline $\mathrm{Co}^{59}(\mathrm{n}, 2 \mathrm{n})$ & $\mathrm{Co}^{58}$ & $4,5 \pm 0,5$ \\
$\mathrm{Se}^{74}(\mathrm{n}, 2 \mathrm{n})$ & $\mathrm{Se}^{73}$ & $5 \pm 0,5$ \\
$\mathrm{Ni}^{58}(\mathrm{n}, \mathrm{p})$ & $\mathrm{Co}^{58}$ & $4 \pm 0,8$ \\
$\mathrm{As}^{75}(\mathrm{n}, \mathrm{p})$ & $\mathrm{Ge}^{75}$ & $5 \pm 0,8$ \\
\hline
\end{tabular}

Tab. 2.

Abb. 6. Experimentelle Ergebnisse über den Spinabschneideparameter $\sigma$.

19 H. K. Vonach, R. Vandenbosch u. J. R. Huizenga, Nucl. Phys. 60, 70 [1964].

20 J. L. NeEd, Phys. Rev. 129, 1302 [1963].

21 W. Patzak u. H. Vonach, Nucl. Phys. 39, 263 [1962].
22 C. E. Hunting, Phys. Rev. 123, 606 [1961].

${ }^{23}$ B. Antolkovic, Nucl. Phys. 44, 123 [1963].

24 A. Zucher, Plenarvorträge, Tagung Deutsche Phys. Ges., Düsseldorf 1964, S. 54. 
parameter $\sigma$ mit dem mittleren Verhalten der übrigen Ergebnisse aus der Literatur zwar überein, jedoch ist insgesamt die Kenntnis über $\sigma$ doch noch sehr unbefriedigend. Die teilweise erheblichen Schwankungen der Werte für $\sigma$ innerhalb enger Bereiche der Kernmassenzahl deuten sehr wahrscheinlich auf noch relativ große Unsicherheiten in den zur Messung von $\sigma$ zur Verfügung stehenden Verfahren hin. Abb. 6 läßt erkennen, daß $\sigma$ im Massenbereich $A=20-80$ von etwa $2,5 \hbar$ auf $5 \hbar$ ansteigt.

Es soll abschließend ein Vergleich der empirischen Werte für $\sigma$ mit den Voraussagen des Fermi-GasModells vorgenommen werden. Dabei ist es zweckmäßig, diesen Vergleich nicht direkt für $\sigma$ durchzuführen, sondern vielmehr das aus $\sigma$ gemäß (2) folgende Trägheitsmoment $\mathcal{T}=\left(\sigma^{2} \hbar^{2}\right) / T$ zu berechnen und mit $\mathcal{T}_{0}$ nach (3) zu vergleichen. Dazu wurde die Kerntemperatur $T$ jeweils nach (4) berechnet, wobei jedoch $E$ durch $E-\delta$ ersetzt wurde, um den Paarungseffekt zu berücksichtigen. Für $E$ wurde $7 \mathrm{MeV}$ gewählt und die Werte für den Parameter $a$ wurden der Arbeit von Erba et al. ${ }^{3}$ entnommen. Die auf diese Weise für die Spinabschneideparameter aus Abb. 6 berechneten Kernträgheitsmomente $\mathcal{T}$ sind in Abb. 7 wiedergegeben. Nach (3) hängt $\mathcal{T}_{0}$ noch vom Kernradius $R$ ab. Es wurde der Ansatz $R=r_{0} A^{1 / 3}$ benutzt und $\mathcal{T}_{\mathbf{0}}$ für die drei verschiedenen Radius- parameter $r_{0}=1,5 ; 1,3$ und $1,2 \mathrm{fm}$ berechnet. Die Ergebnisse sind in Abb. 7 mit eingetragen. Man erkennt, daß $\mathcal{T}_{0}$ etwa eine obere Grenze für die empirischen Werte von $\mathcal{T}$ darstellt, im Mittel liegt jedoch $\mathcal{T}$ unterhalb $\mathcal{T}_{\mathbf{0}}$. Um welchen Prozentsatz $\mathcal{T}$ gegenüber $\mathcal{T}_{0}$ reduziert ist, läßt sich jedoch wegen der starken Streuung der empirischen Daten nicht allgemein angeben; man kann lediglich feststellen, daß stets etwa $\mathcal{T}_{0} / 2 \leqq \mathcal{T} \leqq \mathcal{T}_{0}$ ist. Dieses Ergebnis ist allerdings nicht unerwartet, denn sehr wahrscheinlich liefert das freie Fermi-Gas-Modell bei den hier betrachteten, noch relativ kleinen Anregungsenergien nur ein unvollständiges Bild des Kerns. Insbesondere die Paarungskräfte zwischen den Nukleonen sind bei diesen Energien keinesfalls vernachlässigbar und ihr Einfluß läßt erwarten, daß das wirkliche Kernträgheitsmoment kleiner als das im freien Fermi-GasModell ist ${ }^{19,33}$.

Die Autoren danken besonders Herrn Professor Dr. H. NeuERt für sein Interesse an dieser Arbeit und deren Unterstützung. Herrn Professor Dr. W. Jentschke und Herrn Dr. Sковка sei für die Bereitstellung des $3 \mathrm{MeV}-V_{\text {An de }}$ GraAfF-Generators des II. Instituts für Experimentalphysik der Universität Hamburg ebenfalls gedankt.

Die Untersuchungen wurden teilweise durch das Bundesministerium für wissenschaftliche Forschung unterstützt, wofür wir gleichfalls unseren Dank aussprechen möchten.

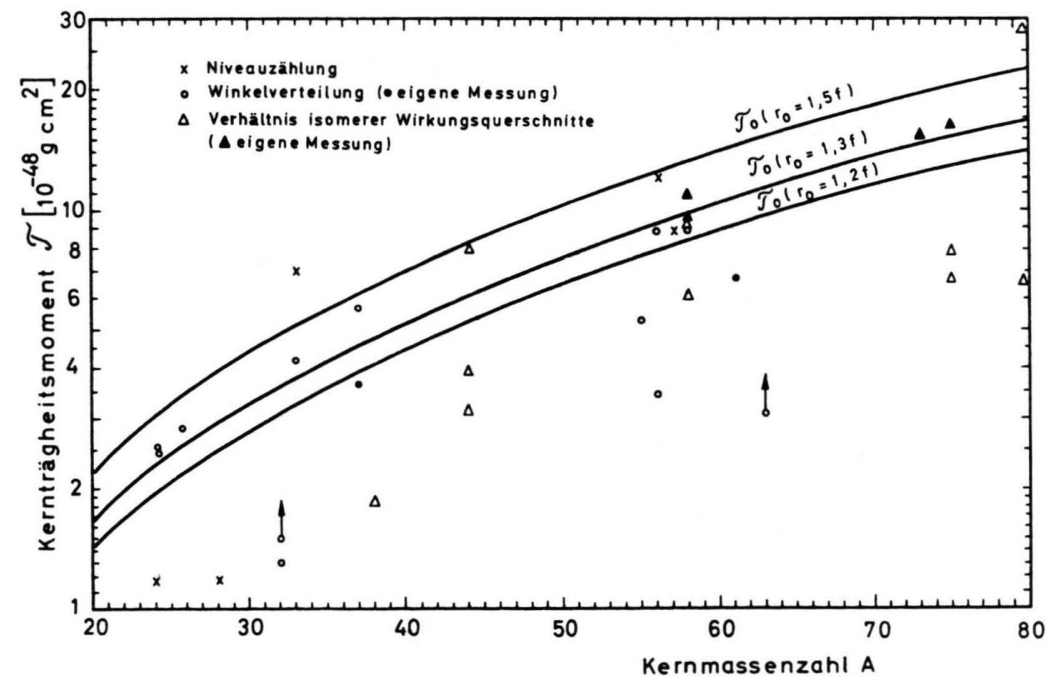

Abb. 7. Experimentelle Ergebnisse über das Kernträgheitsmoment $\mathcal{T}$ und Vergleich mit dem theoretischen Wert $\mathcal{J}_{0}$ (starrer Körper).
25 R. Vandenbosch u. J. R. Huizenga, Phys. Rev. 120, 1313 [1960].

26 E. Weigold u. R. N. Glover, Nucl. Phys. 32, 106 [1962].

27 J. H. Carver, G. E. Cook u. T. R. Sherwood, Nucl. Phys. 37, 449 [1962].

28 F. Fukuzawa, J. Phys. Soc. Japan 16, 2371 [1961].

29 S. K. Mangal u. P. S. Gill, Nucl. Phys. 49, 510 [1963].
30 D. Goldmann, A. F. T. Piza u. E. Silva, Nuovo Cim. 25, 41 [1962].

31 J. R. Tatarczuk u. H. A. Medicus, Int. Conf. „Study of Nuclear Structure with Neutrons", Antwerpen 1965, Bericht 125.

32 S. Chojnacki et al., Int. Conf. „Study of Nuclear Structure with Neutrons", Antwerpen 1965, Bericht 114.

33 D. W. Lang, Nucl. Phys. 42, 353 [1963]. 